\title{
Impact of botanical oils on polyunsaturated fatty acid metabolism and leukotriene generation in mild asthmatics
}

Jonathan P Arm 1,4,5,9, Joshua A Boyce, 4,5, Lin Wang ${ }^{1}$, Heng Chhay ${ }^{1}$, Muhammad Zahid ${ }^{2}$, Vaishali Patil ${ }^{2}$, Usha Govindarajulu', Priscilla Ivester ${ }^{6,7}$, Kelly L Weaver ${ }^{6,10}$, Susan Sergeant ${ }^{7,8}$, Elliot Israel ${ }^{2,3,5}$ and Floyd H Chilton ${ }^{6,7^{*}}$

\begin{abstract}
Background: Dietary supplementation with botanical oils that contain n-6 and n-3 eighteen carbon chain (18C)-PUFA such as $y$ linolenic acid (GLA, 18:3n-6), stearidonic acid (SDA, 18:4n-3) and a linolenic acid (ALA, 18:3n-3) have been shown to impact PUFA metabolism, alter inflammatory processes including arachidonic acid (AA) metabolism and improve inflammatory disorders.
\end{abstract}

Methods: The diet of mild asthmatics patients was supplemented for three weeks with varying doses of two botanical seed oils (borage oil [Borago officinalis, BO] and echium seed oil [Echium plantagineum; EO]) that contain SDA, ALA and GLA. A three week wash out period followed. The impact of these dietary manipulations was evaluated for several biochemical endpoints, including in vivo PUFA metabolism and ex vivo leukotriene generation from stimulated leukocytes.

Results: Supplementation with several EO/BO combinations increased circulating 20-22 carbon (20-22C) PUFAs, including eicosapentaenoic acid (EPA), docosapentaenoic acid (DPA), and dihommo-gammalinolenic acid (DGLA), which have been shown to inhibit AA metabolism and inflammation without impacting circulating AA levels. BO/EO combinations also inhibited ex vivo leukotriene generation with some combinations attenuating cysteinyl leukotriene generation in stimulated basophils by $>50 \%$ and in stimulated neutrophils by $>35 \%$.

Conclusions: This study shows that dietary supplementation with BO/EO alters 20-22C PUFA levels and attenuates leukotriene production in a manner consistent with a reduction in inflammation.

Keywords: Asthma, Gammalinolenic acid, Stearidonic acid, Inflammation, Leukotrienes, Borage oil, Echium oil

\section{Background}

Asthma is a complex disease which involves smooth muscle contraction and inflammation that result in narrowing and obstruction of the airway. Arachidonic acid (AA) metabolism via the 5-lipoxygenease pathway to form leukotrienes has been demonstrated to be particularly important to the pathology of asthma. Specifically, the cysteinyl leukotrienes, $\mathrm{LTC}_{4}, \mathrm{LTD}_{4}$, and $\mathrm{LTE}_{4}$, act at GPCRs, CysLT $_{1} R$ and CysLT $_{2} R$, to elicit their

\footnotetext{
* Correspondence: schilton@wakehealth.edu

${ }^{6}$ Department of Physiology/Pharmacology, Wake Forest School of Medicine, Medical Center Blvd, 27157, Winston-Salem, NC, USA

${ }^{7}$ Center for Botanical Lipids and Inflammatory Disease Prevention, Wake Forest School of Medicine, Medical Center Blvd, 27157, Winston-Salem, NC, USA

Full list of author information is available at the end of the article
}

effects, which include contraction of bronchial smooth muscle, vasodilatation, and mucus secretion within the airways [1,2]. Additionally, $\mathrm{LTB}_{4}$ is a potent chemoattractant for granulocytes, effector $\mathrm{T}$ cells, and monocytes, acting at a specific GPCR, BLT1 [3]. Collectively, leukotrienes have numerous proinflammatory properties, and leukotriene modifying drugs have proven effective in the management of asthma [4] and allergic rhinitis [5].

In addition to pharmacologic modifiers, supplementation of diets with fatty acid-based marine and botanical oil supplements have been demonstrated to reduce the severity of several inflammatory disorders including asthma. One of the primary mechanisms thought to be responsible for their efficacy has been the capacity of $n-6$ and $n-3$ polyunsaturated fatty acids to alter AA metabolism to 
form eicosanoids. For example, dietary supplementation with fish oils that contain eicosapentaenoic acid (EPA; 20:5, n-3) inhibits leukotriene generation, likely through substrate competition of EPA with AA for the action of cytosolic phospholipase $\mathrm{A}_{2}$ and 5-lipoxygenase [6].

In regard to botanical oils, borage seed oils contain the 18 carbon PUFA, gammalinolenic acid (GLA; 18:3, n-6) that is elongated by most tissues and inflammatory cells to dihommo-gammalinolenic acid (DGLA) (Figure 1). Newlyformed DGLA is then incorporated into membrane phospholipids. Like EPA, DGLA has the capacity to compete with AA for eicosanoid production. DGLA is also converted to prostaglandin (PG) $\mathrm{H}_{1}$ via cyclooxygenase enzyme(s), which is then converted to $\mathrm{PGE}_{1}$. $\mathrm{PGE}_{1}$ has been illustrated to have anti-inflammatory effect in both animals and humans [7-9].

In addition, seed oil from another member of the Boraginaceae family, Echium plantagineum contains both n-6 and n-3 18C-PUFAs, including a GLA, $\alpha$-linolenic acid (ALA; 18:3, n-3) and stearidonic acid (SDA; 18:4, n-3). The conversion of ALA to EPA and DHA is poor in humans [10], which is believed to be a result of the inefficiency of the initial rate-limiting step ( $\triangle-6$ desaturase, FADS2 gene) involved in 20-22 carbon PUFA biosynthesis. However SDA is downstream of $\Delta-6$ desaturase and is 4 to 5 -fold more efficiently converted to EPA than ALA [11]. Additionally, SDA has been demonstrated to block in vitro leukotriene generation and in vivo inflammatory processes (reviewed in [11]).
It has been long appreciated that natural products may contain a complex mixture of several active ingredients with synergistic biological effects. As discussed above, botanical seed oils contain several 18C-PUFAs that have the potential to impact diseases that are driven by eicosanoid generation. Several studies have examined the impact of providing individual botanical oils that contain a high proportion of their total fatty acids as a putative PUFA active ingredient; however, little is known regarding the biochemical interactions of adding more than one botanical oil containing several potential PUFA active ingredients. The primary objective of this current study was to examine the biochemical impact of adding PUFAs found in two botanical seed oils on PUFA metabolism and leukotriene generation in mild asthmatic patients.

\section{Materials and methods}

\section{Subjects}

Thirty-seven asthmatic subjects (ages 18-64) were recruited. Written informed consent was obtained from all subjects prior to enrollment. Asthma was diagnosed by the presence of variable airflow obstruction or by a history of treatment for asthma with documented airways hyperresponsiveness to methacholine [12]. Histories (medical and respiratory), a brief physical examination and routine clinical test were used to exclude the presence of significant co-morbid diseases. The inclusion criteria were: 1) male or female 18 years to 65 years of age; 2) asthma with $\mathrm{FEV}_{1} 50$ to $90 \%$ of predicted, or personal best; and 3)

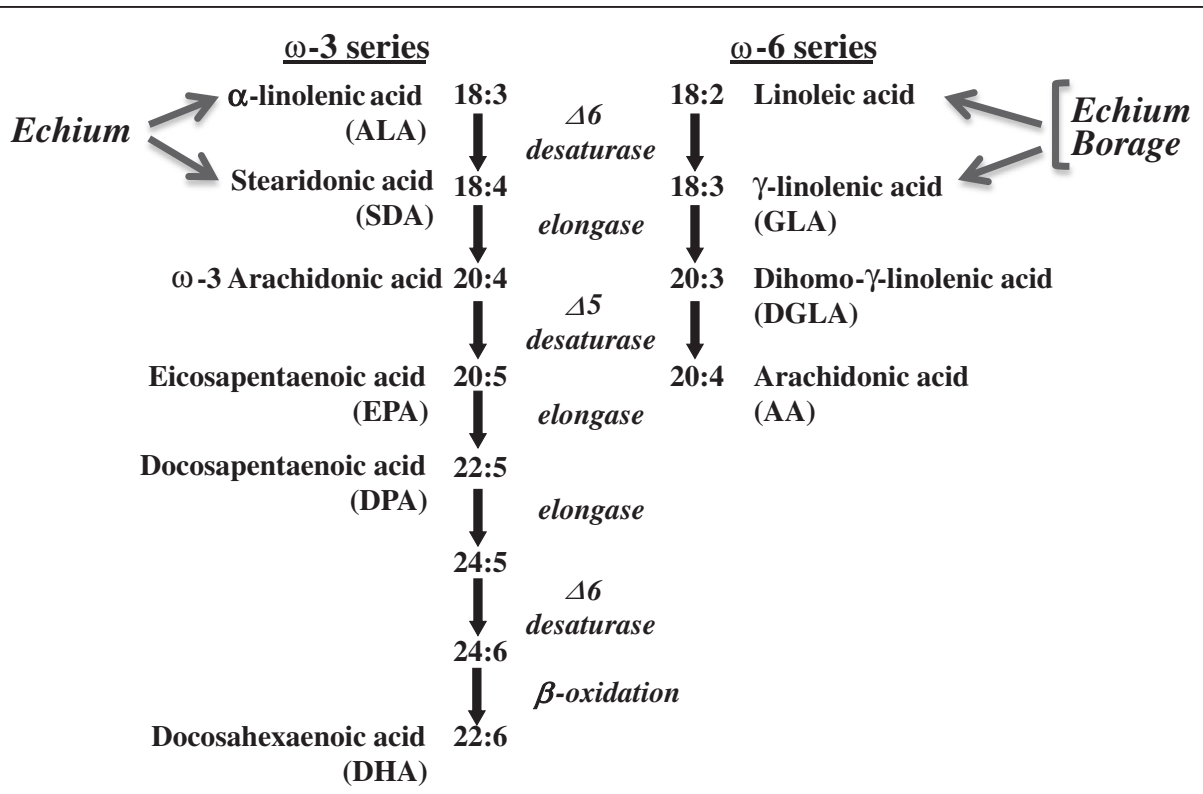

Figure 1 Pathways for metabolism of $\mathbf{n}-\mathbf{6}$ (left) and $\mathbf{n - 3}$ (right) PUFAs in humans. The pathway depicts the synthesis of 20 and 22 carbon PUFAs from the essential, dietary PUFAs, a-linolenic acid ( $n-3)$ and linoleic acid ( $n-6)$. The PUFAs derived from borage oil (linoleic and gammalinolenic acids, both n-6) and echium (a-linolenic, n-3; stearidonic, n-3; linoleic and gamma-linolenic acids, both n-6) would be expected to enter the pathways at the indicated points. 
improvement in $\mathrm{FEV}_{1}>12 \%$ after administration of a beta-2 agonist. The exclusion criteria were: 1 ) pregnant or nursing; 2) smoking history of $>10$ pack years or active smoking within the previous year; 3 ) use of asthma treatments that potentially alter leukotriene biosynthesis including theophylline and oral steroids; 4) dietary supplements with fatty acids or other products that may interfere with leukotriene generation; 5) treatment within the previous three months with omalizumab (monoclonal antibody directed against $\operatorname{IgE}$ ); 6) use of non-steroidal antiinflammatory drugs in the week prior to any measurements of ex vivo leukotriene generation; 7) a history of aspirinsensitive asthma; 8) significant abnormalities in CBC, differential white cell count, renal function, and liver function, or urinalysis; and 9) any serious co-morbid medical condition.

Subjects meeting the entry criteria were randomized to one of the four study groups based on botanical oil dosing (Table 1). Subjects used rescue albuterol less than twice a week, had nocturnal asthma less than twice a month, and had a forced expiratory volume in one second $\geq 75 \%$ predicted. During the month prior to entry into the study, no subject used leukotriene modifiers, oral or high dose inhaled steroids, or theophylline, and

Table 1 Study group compositions and daily dosing scheme for borage and echium seed oils in the four groups of subjects

\begin{tabular}{lllll}
\hline & Group 1 & Group 2 & Group 3 & Group 4 \\
\hline No. of Subjects & 6 & 9 & 11 & 11 \\
(female) & $(3)$ & $(5)$ & $(6)$ & $(7)$ \\
Age (range) & $22-46$ & $18-54$ & $19-58$ & $22-64$ \\
No. of subjects withdrawn & 1 & 1 & 1 & 2 \\
Echium Oil Dose (g) & 14 & 7 & 4 & 2 \\
No. of daily capsules & 14 & 7 & 4 & 2 \\
ALA (g) & 4.02 & 2.01 & 1.15 & 0.57 \\
SDA (g) & 1.75 & 0.875 & 0.5 & 0.25 \\
GLA (g) & 1.54 & 0.77 & 0.44 & 0.22 \\
Borage Oil Dose (g) & 0 & 3.9 & 5.2 & 5.2 \\
No. of daily capsules & 0 & 3 & 4 & 4 \\
ALA (g) & $<0.01$ & $<0.01$ & $<0.01$ & $<0.01$ \\
SDA (g) & 0 & 0 & 0 & 0 \\
GLA (g) & 0 & 0.9 & 1.2 & 1.2 \\
Total & & & & \\
No. of daily capsules & 14 & 10 & 8 & 6 \\
ALA (g) & 4.02 & 2.01 & 1.15 & 0.57 \\
SDA (g) & 1.75 & 0.875 & 0.5 & 0.25 \\
GLA (g) & 1.54 & 1.67 & 1.64 & 1.44 \\
\hline The numbra & & & & \\
\hline
\end{tabular}

The number of daily capsules, containing $1 \mathrm{~g}(\mathrm{EO})$ or $1.3 \mathrm{~g}(\mathrm{BO})$ oil per capsule, and the amount of ALA, SDA and GLA provided by the encapsulated echium and borage oils is shown for each dosing group. Recruitment into Group 1 was stopped after the interim analyses showed that the highest dose of EO was not required to prevent elevations of $A A$ levels in circulating plasma. for three months prior to entry into the study no subject used omalizumab, a monoclonal antibody against IgE. The study was approved by the Partners Human Subjects Research Committee. An investigator-initiated IND was obtained from the Food and Drug Administration (IND number 74,110). Because the outcomes in this study were entirely biochemical with no assessment of clinical efficacy, the study was not registered at clinicaltrials.gov.

\section{Dietary fatty acids}

Capsules containing echium seed oil (1000 mg) or borage seed oil (1300 mg) were supplied by Bioriginal (Saskatoon, SA, Canada). The fatty acid contents of the oils were validated by gas chromatography (Table 2). Since the leaves of Echium plantagineum contain pyrrolizidine alkaloid, an independent assay of this toxin was obtained, assessed by high performance TLC, and found it to be less than $4 \mathrm{ng} / \mathrm{g}$ of oil (Chemisches Laboratorium Dr. Hermann Ulex Nachf, Hamburg-Norderstedt, Germany).

\section{Study design}

The study goal was to test combinations of Boraginaceae family seed oils derived from Borago officinalis (BO) and Echium plantagineum L. (EO) for their capacity to impact circulating PUFA levels and inhibit ex vivo leukotriene generation. After baseline determinations of plasma fatty acid levels and ex vivo leukotriene generation, subjects were randomized to dosing groups (Table 1) ingesting a

Table 2 Fatty acid profile of botanical oils consumed by asthmatic subjects

\begin{tabular}{lll}
\hline & \multicolumn{2}{c}{ \% of Total Fatty Acids } \\
\cline { 2 - 3 } Fatty acid & Echium & Borage \\
\hline C16:0 & 7 & 6.7 \\
C16:1 & 0.2 & 0.2 \\
C18:0 & 4.1 & 3.3 \\
C18:1 & 16.8 & 10.4 \\
C18:2 n-6 & 18 & 24.6 \\
C18:3 n-6 & 10.8 & 40.5 \\
C18:3 n-3 & 28.7 & 0.1 \\
C18:4 n-3 & 12.5 & 0.3 \\
C20:0 & 0.2 & 0.3 \\
C20:1 & 0.7 & 5.2 \\
C20:1 & 0 & 0.3 \\
C22:0 & 0 & 0.6 \\
C22:1 & 0.4 & 3.8 \\
C24:0 & 0 & 0.1 \\
C24:1 & 0 & 3.1 \\
Others & 0.3 & 0.5 \\
Total & 99.7 & 100 \\
\hline
\end{tabular}


constant dose of GLA ( 1.5 g daily), $0.25 \mathrm{~g}$ to $1.75 \mathrm{~g}$ SDA and $0.57 \mathrm{~g}$ to $4.02 \mathrm{~g}$ ALA in divided doses (three times a day) for 3 weeks.

Aside from the addition of supplements, the subject's diets were not altered before or during the study. The 'typical' western diet provides very small quantities of GLA and SDA, and it is difficult to measure these PUFA in circulating or cellular lipids. GLA and SDA are typically only observed when individuals are consuming a GLA- or SDA- containing supplement such as borage, evening primrose, black currant, or echium, and this was an exclusion criterion of the study. ALA (n-3) makes up 1\% of the typical western diet and so the supplement may have some impact on dietary levels. Approximately 7\% of energy in the western diet is LA, and thus the quantities of LA in the supplements would have little total impact on overall linoleic acid (n-6) consumption.

Concentrations of GLA provided to human subjects as borage oil alone have been shown to induce increases in circulating AA levels [13]. It was our hypothesis that the presence of the n-3 18C-PUFA SDA in echium oil would prevent such an increase. However, to assure that there were no elevations of circulating AA out of the normal range, an interim analysis was carried out during the early phase of the study. The interim analyses revealed that AA levels remained constant in all groups and consequently, the study was then completed. Blood and urine were collected weekly for assessment of hematological indices, liver and renal function, plasma fatty acids, ex vivo leukotriene generation. After three weeks of supplementation, subjects entered a 3 week wash-out phase, in which plasma fatty acids and ex vivo leukotriene generation were measured weekly. After dropouts, 6 to 11 individuals completed each arm of the study.

Subjects reported any change in their medical condition at each study visit and used diary cards to record all medical symptoms and to log intake of study oils. Compliance was monitored by medication diaries and counts of returned capsules. These assessments indicated at least $90 \%$ compliance by all subjects and plasma fatty acids measurements, in which no outliers were seen, also reflected in a comparable adherence to the supplementation. As the end points of the study were entirely biochemical, the subjects were not blinded (i.e. subjects took different numbers of capsules depending on the group to which they were assigned). The objective of this biochemical study was to determine the impact of potentially bioactive PUFAs in combinations of $\mathrm{BO}$ and $\mathrm{EO}$ on plasma fatty acid levels and ex vivo leukotriene production. All individuals performing the biochemical assays were blinded to the subjects' study group.

The most common adverse events associated with taking $\mathrm{BO} / \mathrm{EO}$ combinations were gastrointestinal symptoms, occurring in thirteen subjects, variously reported as gas, constipation, loose stools, and/or abdominal pain or discomfort. With the exception of the 3 subjects who withdrew due to gastrointestinal discomfort (Groups 2, 3 and $4)$, these symptoms were mild and transient, occurred in the first few days of the study and resolved within 2 to 3 days while still taking BO/EO combinations. Other adverse events included cold-like symptoms $(n=7$ : Group 1 $\mathrm{n}=3$; Group 2, $\mathrm{n}=2$; Group 3, $\mathrm{n}=1$; Group 4, $\mathrm{n}=1$ ), pharyngitis ( $n=2$; one each in Groups 2 and 4), mild symptoms of asthma associated with seasonal allergies $(n=2$, one each in Groups 3 and 4), transient wrist pain ( $\mathrm{n}=1$; group 3), and transient itchy rash $(n=1$; Group 4). Two other subjects withdrew from the study due to the development of itch and mild asthma flare (Group 4) and unforeseen extension of an out-of-town business trip (Group 1). There were no significant changes in vital signs in any study subjects.

There were no consistent or significant changes in biochemical laboratory parameters or urinalysis during the study (data not shown). However, in four of the individuals who entered the study ( 2 male, 2 female) there was a decrease in circulating hemoglobin of $>1.0 \mathrm{~g} / \mathrm{dl}$. Across all study groups there was on average a decrease in circulating hemoglobin of $0.45 \mathrm{~g} / \mathrm{dl}(13.8$ to $13.3 \mathrm{~g} / \mathrm{dl}$; p < 0.001$)$, which did not vary significantly between study groups ( $\mathrm{p}=0.09$ for differences among groups across time). In three individuals, the value dipped just below the range of normal. There was an accompanying decrease in red cell count from 4.58 to $4.39 \times 10^{12}$ /liter with no change in mean corpuscular volume. A decrease in hemoglobin concentration was not seen in all subjects. There were no accompanying decreases in white cell count or platelet count.

\section{Plasma and botanical oil fatty acids analysis}

Fatty acid methyl esters were prepared in duplicate plasma samples $(100 \mu \mathrm{l})$ following a modification of Metcalfe et al. [14] and analyzed by gas chromatography as previously described [15]. Encapsulated oils were suitably diluted in hexane and submitted to fatty acid analysis in a manner comparable to that for plasma. Fatty acids in sample were identified based on retention times of authentic fatty acid methyl esters (Supleco, Bellefonte, PA, USA; Cayman Chemicals, Ann Arbor MI, USA; Matreya, Pleasant Gap PA, USA; NuChek Prep, Elysian, MN, USA).

\section{Ex vivo leukotriene generation}

FcERI-dependent generation of cysteinyl leukotrienes from basophils. Peripheral blood mononuclear cells (PBMC), typically containing $\sim 3$ to $4 \%$ basophils, were isolated from $20 \mathrm{~mL}$ of blood by density gradient centrifugation over Percoll (GE Healthcare, Piscataway, NJ) as described [16]. As previously described [16], basophils 
were stimulated in PBMC by cross-linking of the high affinity Fc receptor for IgE (FceRI). Under these circumstances, the basophil is the predominant, if not the only, source of cysteinyl leukotrienes. Briefly, cells were primed with $10 \mu \mathrm{g} / \mathrm{L}$ IL-3 on ice and stimulated with 0.01 to $1.0 \mu \mathrm{g} / \mathrm{mL}$ of $15 \mathrm{~A} 5$, an activating monoclonal antibody to FceRI, for $30 \mathrm{~min}$ at $37^{\circ} \mathrm{C}$. Cysteinyl leukotrienes in the supernatant fluids were stored at $-80^{\circ} \mathrm{C}$ until assayed by immunoassay (Cayman Chemical Company, Ann Arbor, MI or GE Healthcare) for $\mathrm{LTC}_{4}$ with $100 \%$ cross-reactivity with $\mathrm{LTC}_{5}, 48 \%$ and $46 \%$ cross-reactivity with $\mathrm{LTD}_{4}$ and $\mathrm{LTD}_{5}$, respectively, and $<10 \%$ crossreactivity with $\mathrm{LTE}_{4}$ and $\mathrm{LTE}_{5}$.

A23187-stimulated generation of leukotrienes from neutrophils. Neutrophils were isolated from heparinized blood by dextran sedimentation, density gradient centrifugation through Ficoll Paque (GE Healthcare, Uppsala, Sweden), and hypotonic lysis of contaminating red cells [17]. Neutrophils $\left(2 \times 10^{6}\right)$ were stimulated $\left(5 \mathrm{~min}, 37^{\circ} \mathrm{C}\right)$ with 0.1 to $10 \mu \mathrm{mol} / \mathrm{L}$ A23187 (Sigma-Aldrich, St Louis, MO) in Hanks buffered salt solution containing $1.25 \mathrm{mmol} / \mathrm{L} \mathrm{cal-}$ cium and magnesium salts, $25 \mathrm{mmol} / \mathrm{L}$ Hepes, and $1 \mathrm{~g} / \mathrm{L}$ fatty acid-free bovine serum albumin. Leukotrienes in the supernatants were resolved by reverse-phase HPLC (RPHPLC) as described [18] and quantified by absorbance at $280 \mathrm{~nm}\left(\mathrm{LTB}_{4}\right.$ and all-trans $\left.\mathrm{LTB}_{4}\right)$ and $235 \mathrm{~nm}$ (5hydroxyeicosatetraenoic acid, 5-HETE).

\section{Statistical analysis}

Statistical analyses were performed using SAS Version 9.2. We employed a repeated measures mixed model (rmm) analysis for each fatty acid to predict the response in which repeated measures were taken on each subject at each visit (time) and the group and time interactions were modeled along with the main effects. The model utilized a compound symmetry correlation structure since the correlation between pairs of times would be similar and not expected to change across time. For analyses of ex vivo leukotriene generation by neutrophils and basophils, we also considered the effect of a range of doses of each stimulus at each visit within the same modeling. We then used adjusted least square mean differences obtained from each rmm model to compare the differences between groups and between times within a single group. All analyses were conducted at a 0.05 level of significance.

\section{Results}

\section{Plasma fatty acids}

The effects of dietary supplementation with $\mathrm{BO}$ and EO on n-3 and n-6 PUFA levels are provided in Figure 2 and Figure 3, respectively. ALA (18:3n-3), present in echium oil, tended to increase in all groups during the supplementation period (weeks 1-3) and was significantly higher
( $\mathrm{p}<0.05$, Figure 2, white bar) than baseline in Group 1 (wk 3 ) and Group 3 (wk 2). SDA (18:4n-3) was not detectable in the plasma at baseline but rose significantly during supplementation with BO and EO $(\mathrm{p}<0.0001$, Figure 2, black bar). As expected, the increase in plasma SDA varied between groups and was most marked in Group 1 in which subjects consumed the largest dose of echium oil ( $\mathrm{p}=0.004$ for differences among groups across time). In each group, the plasma levels of EPA (20:5n-3; Figure 2, gray bar) and its elongation product DPA (22:5n-3; Figure 2, cross-hatched bar) rose significantly $(\mathrm{p}<0.0001$ for each fatty acid) in a manner that was dependent on the dose of echium oil ingested ( $p=0.004$ for group by time interactions for each fatty acid). However, there was no rise in circulating plasma DHA (22:6n-3; Figure 2, white striped bar) in any group $(p=0.14)$. During the washout phase, plasma SDA, EPA and DPA rapidly returned to baseline levels.

With respect to n- 6 fatty acids, plasma concentrations of GLA (18:3n-6; Figure 3, black bar) and DGLA (20:3n-6; Figure 3 , white bar) rose in each group ( $<0.0001$ for each fatty acid) with no significant difference between groups $(\mathrm{p}=0.06$ and $\mathrm{p}=0.63$, respectively, for differences among groups across time). Importantly, concentrations of plasma AA (20:4n-6; Figure 3, gray bar) remained constant throughout with no significant change during supplementation with $\mathrm{BO}$ and $\mathrm{EO}$ in any group $(\mathrm{p}=0.39$ for effect of time, and $\mathrm{p}=0.87$ for group by time interactions). During the washout phase, plasma GLA and DGLA rapidly returned to baseline levels.

\section{Ex vivo leukotriene generation}

Figure 4 illustrates the effect of dietary supplementation with a constant dose of $\mathrm{BO}$ and variable doses of $\mathrm{EO}$ on ex vivo cysteinyl leukotriene generation from basophils stimulated through FceRI. Basophils are the only cell among peripheral blood mononuclear cells that express the fully functional heterotetrameric $\alpha \beta \gamma_{2}$ form of FceRI and are the principal, if not the only, source of cysteinyl leukotrienes when this population of cells is stimulated through this receptor [16]. We therefore stimulated basophils at $\sim 3$ to $4 \%$ purity in PBMC with an activating antibody to FceRI, before (baseline) and at weekly intervals for three weeks while subjects supplemented their diet with borage and echium seed oils. Because of the marked difference among individuals in absolute quantities of cysteinyl leukotrienes generated by basophils, varying from 1 to $90 \mathrm{ng} / 10^{6}$ basophils, data are expressed as a percentage of maximal leukotriene generation per million basophils prior to treatment with $\mathrm{BO}$ and EO. Compared to baseline leukotriene generation (Figure 4, closed circles), supplementation with BO and EO resulted in decreased FceRI-mediated leukotriene generation. This inhibitory effect was statistically significant in all groups during 


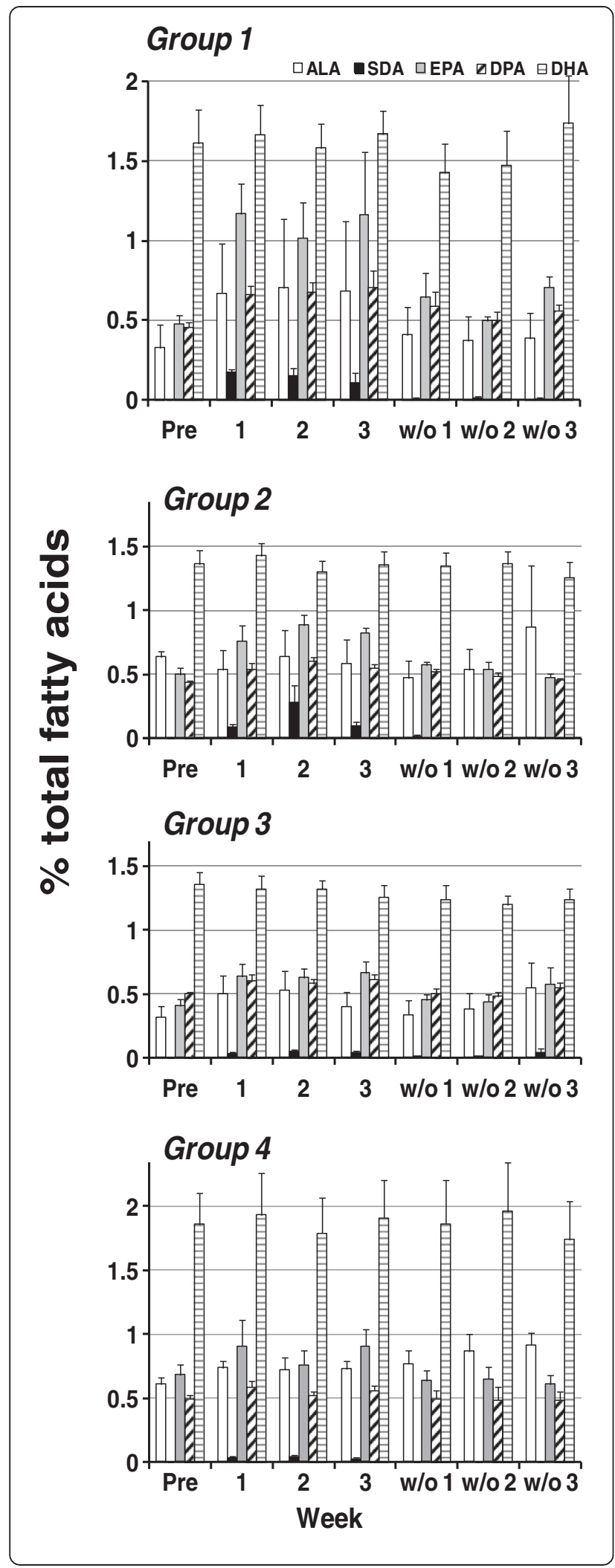

Figure 2 Effects of dietary supplementation with borage and echium seed oils on concentrations of plasma n-3 fatty acids. Data are expressed as a percentage of total plasma fatty acids and are shown for ALA (open bar), SDA (black bars), EPA (gray bars), DPA (cross-hatched bars), and DHA (white striped bars). Fatty acid profiles were monitored during the time course of the study beginning at baseline (Pre), during supplementation (weeks 1-3) and during the washout phase (w/01-3). Data are mean \pm SEM for each group. Statistically significant rises in SDA, EPA and DPA were noted over time $(p<0.0001)$ with significant differences among groups $(p=0.004)$.

treatment with $\mathrm{BO}$ and $\mathrm{EO}(\mathrm{p}<0.0001)$ with a significant difference between study groups $(p<0.0001$ for differences among groups across time). The most stable and robust inhibition of leukotriene generation was seen in Group 2 (Figure 4), in which individuals ingested EO/BO combination that provided $1.7 \mathrm{~g}$ of GLA, $2.01 \mathrm{~g}$ ALA and 0.9 g SDA daily. In this group there was a time-dependent inhibition of cysteinyl leukotriene generation from basophils, stimulated with all concentrations of the antibody to FceRI that reached $57 \%$ and $50 \%$ inhibition of the maximal response by weeks 2 and 3, respectively. A similar inhibition of FceRI-dependent cysteinyl leukotriene generation was observed in Group 3 (Figure 4) in which individuals ingested a $\mathrm{BO} / \mathrm{EO}$ combination that provided $1.6 \mathrm{~g}$ of GLA, $1.15 \mathrm{~g}$ ALA and $0.5 \mathrm{~g}$ SDA daily $(\mathrm{n}=8)$. In Group 3, inhibition was maximal $(60 \%)$ at week one and cysteinyl leukotriene generation remained suppressed at weeks 2 and 3. Inhibition of FceRI-dependent cysteinyl leukotriene generation from peripheral blood basophils was less robust in Groups 1 and 4.

The inhibitory effect of dietary BO and EO on basophilic leukotriene generation was transient. Figure 5 shows the reversal of ex vivo cysteinyl leukotriene generation from basophils (stimulated with a maximum concentration of stimulating antibody) during the washout period after individuals stopped taking capsules of borage and echium oil supplements. Data are expressed as percentage of the maximum cysteinyl leukotriene generation prior to starting supplements. In all groups, there was a return to baseline values within 3 weeks.

A23187 is a calcium ionophore that stimulates leukotriene generation by eliciting calcium flux in a receptorindependent manner. In neutrophils, it provides a robust stimulus for leukotriene generation and allows assessment of the integrity of the whole leukotriene biosynthetic pathway including the terminal product, $\mathrm{LTB}_{4}$, and the stable non-enzymatic degradation products of its intermediates, 5-HETE (from 5-hydroperoxyeicosatetraenoic acid) and all-trans-LTB 4 (from $\mathrm{LTA}_{4}$ ). We therefore examined the effects of dietary supplementation with $\mathrm{BO}$ and $\mathrm{EO}$ on A23187-stimulated leukotriene generation from neutrophils. The shape and position of the dose response curves for release of $\mathrm{LTB}_{4}$ and 5-HETE were similar at baseline, 


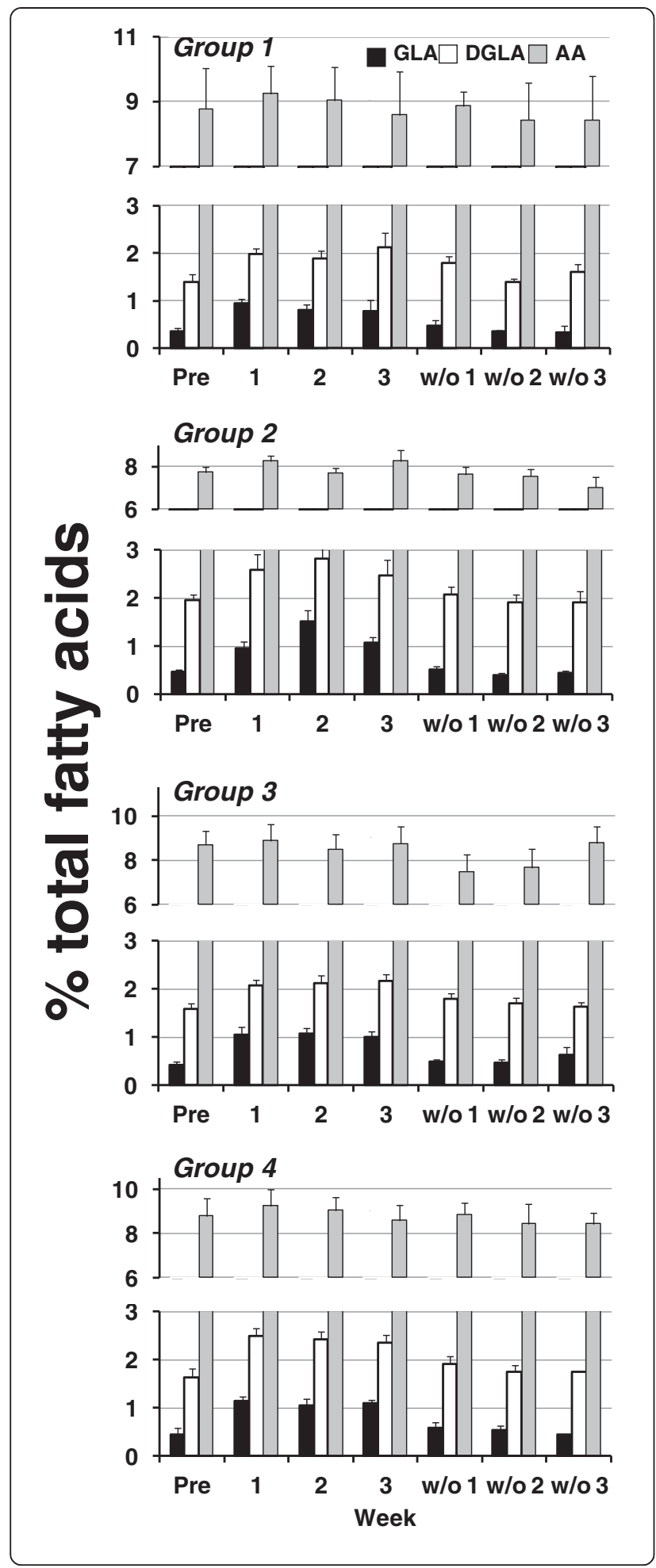

Figure 3 Effects of dietary supplementation with borage and echium seed oils on concentrations of plasma n-6 fatty acids. Data are expressed as a percentage of total plasma fatty acids and are shown for GLA (black bars), DGLA (white bars), and AA (gray bars). Fatty acid profiles were monitored during the time course of the study beginning at baseline (Pre), during supplementation (weeks 1-3) and during the washout phase (w/01-3). Data are mean \pm SEM for each group. Statistically significant rises in GLA and DGLA were noted over time $(p<0.0001)$ with no statistically significant differences among groups.

and changes in generation of $\mathrm{LTB}_{4}$ and 5-HETE during dietary supplementation with $\mathrm{BO}$ and $\mathrm{EO}$ were similar (data not shown). Therefore, data are presented as total leukotriene generation; i.e. the sum of 5-HETE, $\mathrm{LTB}_{4}$, and all-trans-LTB ${ }_{4}$ A23187 elicited the dose-dependent release of leukotrienes that was apparent at a concentration of $0.3 \mu \mathrm{M}$ and reached a maximum response at a concentration of 3 to $10 \mu \mathrm{M}$ A23187 (Figure 6). The quantities of all-trans $\mathrm{LTB}_{4}$ diastereoisomers that were generated were small and were not always readily measured by UV absorbance; they are included in the data where possible. Treatment with $\mathrm{BO} / \mathrm{EO}$ combinations led to a significant inhibition of A23187-stimulated leukotriene generation from neutrophils $(\mathrm{p}<0.0001)$ with a significant difference in effect between groups $(\mathrm{p}=0.02$ for differences among groups across time). The group-dependent effects of dietary supplementation with BO and EO on A23187stimulated leukotriene generation from neutrophils were similar to those observed for FceRI-dependent cysteinyl leukotriene generation from basophils (Figure 4). The greatest effect was seen in Group 2 (Figure 6), in which maximal total leukotriene generation was inhibited $43 \%$ after three weeks of dietary supplementation with $\mathrm{BO} / \mathrm{EO}$ combinations. The inhibitory effect of the dietary supplementation on neutrophil leukotriene generation declined over time in Group 3 and was less robust in Group 4 (Figure 6). As in the case of basophils, A23187-stimulated leukotriene generation from neutrophils returned to baseline during the 3-week wash-out period (data not shown).

\section{Discussion}

Botanical seed oils from plants such as borage and echium have shown modest efficacy in a number of animal and human inflammation models and disease. These botanicals contain 18C-PUFAs (ALA, SDA and GLA) that can be metabolized into 20-22 carbon PUFAs such as EPA, DHA, DGLA and AA. All of these have been shown to impact eicosanoid generation. However, a better understanding of the in vivo biochemistry of potentially bioactive PUFAs found in these botanical seed oils and oil combinations and their capacity to block inflammatory processes including eicosanoid production is needed to enhance the effectiveness of botanical seed 


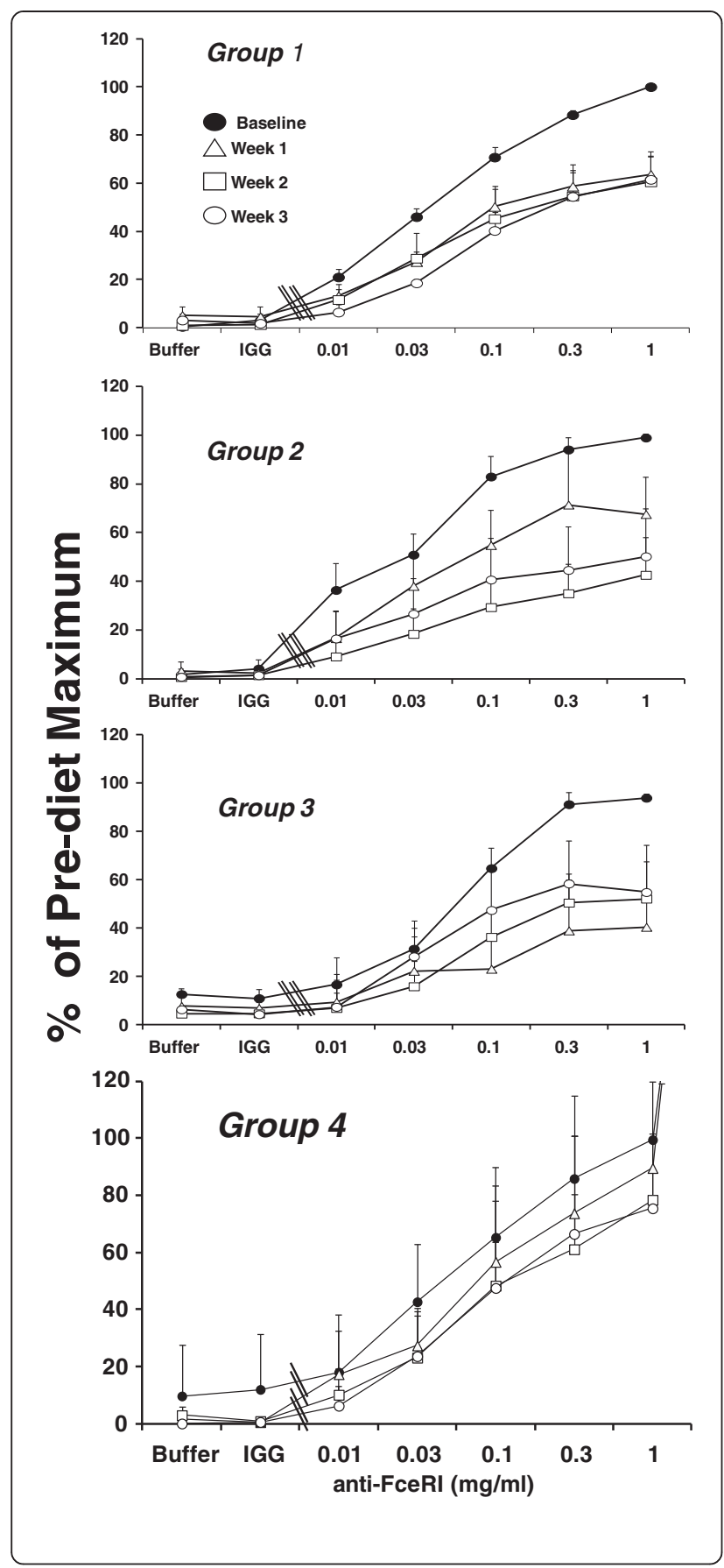

Figure 4 Dietary supplementation with borage and echium seed oils decreases Fc\&RI-dependent cysteinyl leukotriene generation by peripheral blood basophils. Cysteinyl leukotriene generation is shown prior to $(\bullet$, closed circles) and one week $(\triangle$, open triangles), two weeks ( $\square$, open squares), and three weeks ( 0 , open circles) after dietary supplementation with one of four borage and echium seed oil combinations (Groups 1-4, respectively) in response to buffer alone, control $\lg G 1(1.0 \mu \mathrm{g} / \mathrm{ml}, \operatorname{lgG})$, and 15A5, an activating antibody against FcعRl $(0.01$ to $1.0 \mu \mathrm{g} / \mathrm{ml})$. Due to technical errors, data are not available for one subject in each of groups 1, 3, and 4. In addition, basophils from one subject in each of groups 3 and 4 failed to release leukotrienes upon stimulation, a well described phenomenon due to impaired signaling through Syk. Data are expressed as a percentage of maximal cysteinyl leukotriene generation in each subject and are expressed as means \pm SEM. Statistically significant suppression of ex vivo leukotriene generation was noted $(p<0.0001)$ with a significant difference between groups $(p<0.0001)$

oils. The current study utilized various $\mathrm{BO} / \mathrm{EO}$ combinations to understand these processes.

Supplementation with $\mathrm{BO} / \mathrm{EO}$ combinations increases plasma levels of $n-3$ and n-6, 18 carbon and 20-22 carbon PUFAs during the supplementation periods (Figure 2 and Figure 3). Of note, circulating levels of three PUFAs, DGLA, EPA and DPA increased after supplementation. It is likely that DGLA increased as a result of GLA found in both $\mathrm{BO}$ and $\mathrm{EO}$. As discussed above, GLA is readily elongated to DGLA in cells and tissues utilizing an enzyme encoded for by a gene known as elongase 5 (ELOVL5). Once formed, DGLA is incorporated into inflammatory cells and tissues and competes with AA for the action of cytosolic phospholipase $\mathrm{A}_{2}$ and cyclooxygenase to

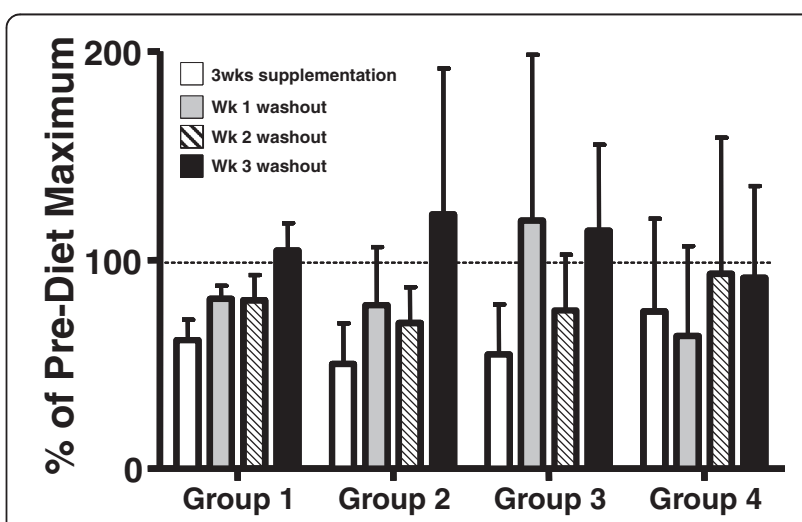

Figure 5 Cessation of dietary supplementation reverses inhibition of Fc\&RI-dependent cysteinyl leukotriene generation by peripheral blood basophils. After 3 weeks of dietary supplementation (open bars), subjects stopped ingesting borage and echium seed oils. FceRl-stimulated basophilic cysteinyl leukotriene generation was measured at one (gray bar), two (cross-hatched bar) and three weeks (solid bar) of washout. Data are shown for maximal cysteinyl leukotriene generation in response to $1.0 \mu \mathrm{g} / \mathrm{ml}$ 15A5. Data are expressed as a percentage of maximal cysteinyl leukotriene generation in each subject and are expressed as means $\pm \mathrm{SEM}$. 


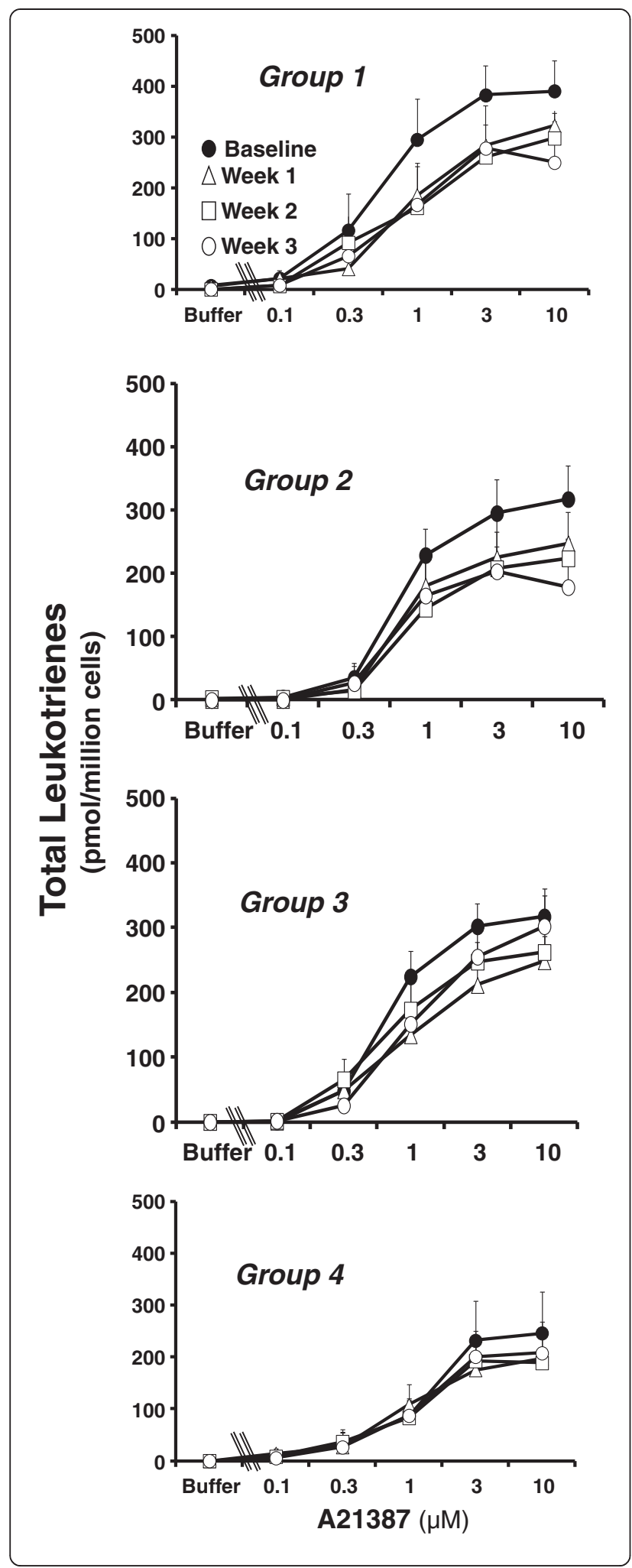

Figure 6 Dietary supplementation with borage and echium oils decreases A23187-stimulated total leukotriene generation by peripheral blood neutrophil. Leukotriene generation is shown prior to $(\bullet$, closed circles) and one week $(\Delta$, open triangles), two weeks ( $\square$, open squares), and three weeks (o, open circles) after dietary supplementation with one of four borage and echium seed oil combinations (Groups 1-4, respectively) in response to buffer alone and increasing concentrations of the calcium ionophore, A23187 $(0.1$ to $10 \mu \mathrm{M})$. Total leukotriene generation is the sum of $\mathrm{LTB}_{4}$, 5-HETE, and, where measurable, all-trans-LTB 4 isomers. Data are expressed as means \pm SEM. Statistically significant suppression of ex vivo leukotriene generation was noted $(p<0.0001)$ with a significant difference in effect between groups $(p=0.02)$.

form $\mathrm{PGE}_{1}$. Additionally DGLA is converted to a 15lipoxygenase product, 15-hydroxyeicosatrienoic acid (15 HeTrE) by human mononuclear leukocytes [18]. 15-HETrE has been demonstrated to be a potent blocker of $\mathrm{LTB}_{4}$ formation.

Additionally this botanical oil combination increased circulating levels of EPA and DPA This contribution was like due to $\mathrm{EO}$ addition since it contains the precursor PUFAs, ALA and SDA. Providing $0.25 \mathrm{~g} / \mathrm{d}$ to $1.75 \mathrm{~g} / \mathrm{d}$ of SDA and $0.57 \mathrm{~g} / \mathrm{d}$ to $4.02 \mathrm{~g} / \mathrm{d}$ of ALA from EO led to significant and dose-dependent increases in circulating EPA and DPA; plasma EPA concentrations rose more than 2-fold in the group receiving the highest concentration of SDA (Figure 2). This increase in EPA and DPA is likely a result of SDA and not ALA as in vivo SDA conversion to EPA is 4-5 fold more efficient than ALA. However, some epidemiological studies suggest that ALA-containing oils (from seed oils such as flax; Linum usitatissimum L.) [19] may provide independent protection from cardiovascular disease.

Numerous studies show the biological impact of EPA and DPA. EPA reduces AA metabolism through several mechanisms including decreasing AA mobilization from membrane phospholipids, competition for cylooxygenase and 5-lipoxygenease and reducing the expression of AA metabolizing enzymes and proinflammatory cytokines. Additionally, EPA can serve as a substrate for prostaglandin formation generating "3-series" prostaglandin products including $\mathrm{PGD}_{3}, \mathrm{PGE}_{3}, \mathrm{PGF}_{3 \alpha}, \mathrm{PGI}_{3}$, and $\mathrm{TxA}_{3}$ and "5 series" leukotriene products including $\mathrm{LTB}_{5}$ and $\mathrm{LTC}_{5}$ [20]. Reduced asthma symptoms with $\mathrm{n}-3$ PUFA ingestion have been shown to be related to 5-series leukotriene production [21]. With regard to inflammation, DPA is beginning to receive attention. DPA is converted to 11hydroxy-7,9,13,16,19-DPA and 14-hydroxy7,10,12,16,19DPA, which inhibit aggregation of platelets and contain 10-fold greater capacity to elicit endothelial cell migration than EPA, a biological process critical to wound healing $[22,23]$. There were no changes in plasma levels of DHA, likely reflecting the poor bioconversion of EPA to DHA. 
Previous studies have shown that GLA-containing oils such as BO have the potential to increase circulating AA which could enhance inflammation and platelet aggregation through increased thromboxane formation [24]. However, there were no changes in AA levels as a result of $\mathrm{BO} / \mathrm{EO}$ supplementation. It is possible that the observed increase in EPA resulting from of the botanical combination is a feedback inhibitor of AA production via the $\Delta 5$ desaturation step. EPA has been demonstrated to inhibit the in vivo and in vitro desaturation of DGLA to form AA $[25,26]$. In any event, the BO/EO combination led to an increase in three 20-22 carbon PUFAs, DGLA, EPA and DPA that have been demonstrated to inhibit AA metabolism and attenuate inflammation without increasing circulating levels of AA.

The final objective of this paper was to determine whether these botanical oil combinations had the capacity to inhibit leukotriene generation from two inflammatory cells, basophils and neutrophils, isolated from subjects with mild asthma who had supplemented their diet with $\mathrm{BO} / \mathrm{EO}$. Prior studies of dietary supplementation with GLA have demonstrated a reduction in ex vivo leukotriene generation in whole blood or neutrophils stimulated with calcium ionophore A23187 or with zymosan [13,26,27]. Basophils [28], IgE [29], and cysteinyl leukotrienes [30] have been strongly implicated in the pathobiology of asthma. We therefore assessed the effects of BO/EO combinations on the generation of cysteinyl leukotrienes from basophils stimulated through the high affinity IgE receptor, a physiologically relevant stimulus for asthma. Significant inhibition of basophil cysteinyl leukotriene generation was noted within one week of dietary supplementation (Figure 4). Interestingly, the time dependence of this inhibition varied between groups. The least robust inhibition was observed in Group 4, in which subjects received the lowest dose of SDA. Although there is considerable variation in the extent of inhibition of ex vivo leukotriene generation among individuals and the groups were relatively small, the between group variation was statistically significant. Furthermore, a comparable variation in supplementation-induced inhibition of leukotriene generation was observed in response to A23187- stimulation of neutrophils (Figure 6). The data therefore suggest that providing SDA in the diet contributed to the extent of inhibition of leukotriene generation, consistent with data showing that dietary supplementation with EPA leads to inhibition of ex vivo leukotriene generation [31-33].

In a receptor-independent manner, A23187 robustly stimulates human neutrophils to elicit maximal generation of $\mathrm{LTB}_{4}$, the product of leukotriene biosynthesis in neutrophils. Utilization of this stimulus allows an assessment by RP-HPLC of the non-enzymatic degradation products of the proximal intermediates of leukotriene biosynthesis. Dietary supplementation with $\mathrm{BO} / \mathrm{EO}$ combinations led to a significant inhibition of A23187stimulated leukotriene generation (Figure 6) that was almost as great as the inhibition of basophil cysteinyl leukotriene generation (Figure 4). We recently reported that dietary supplementation with $\mathrm{BO}$ and fish oil led to reduced expression of phosphatidylinositol 3-kinase, a key signaling molecule, in circulating mononuclear cells [15]. It is therefore possible that the inhibition of cysteinyl leukotriene generation that we observed in basophils was due, at least in part, to inhibition of signaling through FceRI. However, the inhibition of leukotriene biosynthesis in neutrophils stimulated through A23187, a receptor independent stimulus, argues for a more direct effect of BO and EO on leukotriene biosynthesis.

\section{Conclusion}

Our data demonstrate that ingestion of certain combinations of BO and EO increases circulating levels of both n-6 and n-3, 20-22 carbon PUFAs. Supplementation of human and animal diets with these fatty acids has been shown to inhibit metabolism of AA to pro-inflammatory lipid mediators and ameliorate inflammatory disease models including asthma, arthritis and coronary artery disease [34]. These same combinations do not increase circulating AA levels, as has been observed with BO alone. Importantly, the observed biochemical changes occur concomitantly with a reduced capacity of inflammatory cells from mild asthmatics supplemented with $\mathrm{BO} / \mathrm{EO}$ combinations to produce leukotrienes that have been illustrated to be important to promoting the asthmatic response. Consequently, an important next step would be to determine whether such $\mathrm{BO} / \mathrm{EO}$ combinations have the capacity to improve clinical symptoms of asthma.

\section{Abbreviations}

AA: Arachidonic acid; ALA: a linolenic acid; BLT1: B leukotriene receptor 1; BO: Borage oil; Cys $L T_{1} R$ : Cysteinyl leukotriene receptor 1; Cys $L T_{2} R$ : Cysteinyl leukotriene receptor 2; DGLA: Dihommo-gammalinolenic acid;

DHA: Docosahexaenoic acid; DPA: Docosapentaenoic acid; EO: Echium oil; EPA: Eicosapentaenoic acid; GLA: $\gamma$ linolenic acid; GPCR: G protein coupled receptor; IND: Investigational new drug; $\mathrm{LTB}_{4}$ : Leukotriene $\mathrm{B}_{4}$; $\mathrm{LTC}_{4}$ : Leukotriene $\mathrm{C}_{4} ; \mathrm{LTD}_{4}$ : Leukotriene $\mathrm{D}_{4} ; \mathrm{LTE}_{4}$ : Leukotriene $\mathrm{E}_{4}$ : PBMC: Peripheral blood mononuclear cells; SDA: Stearidonic acid.

\section{Competing interests}

FHC is an unpaid consultant for Gene Smart Health and receives no compensation or equity in this role. This information has been disclosed to WFUHS and outside sponsors, as appropriate, and is institutionally managed. All other authors declare no competing interest.

\section{Authors' contributions}

JPA and FHC designed the study and protocols, oversaw the conduct of the study and wrote the manuscript; El participated in the conduct of the study; LW and HC performed ex vivo experiments and sample analyses; PI and KLW analyzed fatty acids and biomarkers; MZ and VP were study coordinators; UG performed statistical analyses; JAB and SS helped with data analysis and interpretation and manuscript preparation. All authors read and approved the final manuscript. 


\section{Acknowledgements}

Supported by a grant from the National Institutes of Health, P50 AT002782, a bridge grant from the American Academy of Allergy Asthma and Immunology and the Fund to Sustain Research Excellence, Brigham and Women's Hospital Biomedical Research Institute.

\section{Author details}

'Division of Rheumatology, Immunology and Allergy, Brigham and Women's Hospital, 75 Francis St 02115, Boston, MA, USA. ${ }^{2}$ Pulmonary Division, Brigham and Women's Hospital, 75 Francis St 02115, Boston, MA, USA. ${ }^{3}$ Center for Clinical Investigation, Brigham and Women's Hospital, 75 Francis St 02115, Boston, MA, USA. ${ }^{4}$ Partners Asthma Center, Brigham and Women's Hospital, 75 Francis St 02115, Boston, MA, USA. ${ }^{5}$ Department of Medicine, Harvard Medical School, 25 Shattuck Street, 02115, Boston, MA, USA. ${ }^{6}$ Department of Physiology/Pharmacology, Wake Forest School of Medicine, Medical Center Blvd, 27157, Winston-Salem, NC, USA. ${ }^{7}$ Center for Botanical Lipids and Inflammatory Disease Prevention, Wake Forest School of Medicine, Medical Center Blvd, 27157, Winston-Salem, NC, USA. ${ }^{8}$ Department of Biochemistry, Wake Forest School of Medicine, Medical Center Blvd, 27157, Winston-Salem, NC, USA. ${ }^{9}$ Current address: Novartis International AG, CH-4002, Basel, Switzerland. ${ }^{10}$ Current address: Department of Surgery, University of Miami School of Medicine, 1600 NW 10th Ave, 33136, Miami, FL, USA.

Received: 23 May 2013 Accepted: 26 September 2013 Published: 2 October 2013

\section{References}

1. Heise CE, O'Dowd BF, Figueroa DJ, Sawyer N, Nguyen T, Im DS, Stocco R, Bellefeuille JN, Abramovitz M, Cheng R, et al: Characterization of the human cysteinyl leukotriene 2 receptor. J Biol Chem 2000, 275:30531-30536.

2. Lynch KR, O'Neill GP, Liu Q, Im DS, Sawyer N, Metters KM, Coulombe N, Abramovitz M, Figueroa DJ, Zeng Z, et al: Characterization of the human cysteinyl leukotriene CysLT1 receptor. Nature 1999, 399:789-793.

3. Henz BM, Jabolonska S, van de Kerhof PC, Stingl G, Blazczyk M, Vandervalk $P G$, Veenhuizen R, Muggli R, Raederstorff D: Double-blind, multicentre analysis of the efficacy of borage oil in patients with atopic eczema. Br J Dermatol 1999, 140:685-688.

4. Malmstrom K, Rodriguez-Gomez G, Guerra J, Villaran C, Pineiro A, Wei LX, Seidenberg BC, Reiss TF: Oral montelukast, inhaled beclomethasone, and placebo for chronic asthma. Ann Intern Med 1999, 130:487-495.

5. Philip G, Malmstrom K, Hampel FC, Weinstein SF, LaForce CF, Ratner PH, Malice MP, Reiss TF: Montelukast for treating seasonal allergic rhinitis: a randomized, double-blind, placebo-controlled trial performed in the spring. Clin Exp Allergy 2002, 32:1020-1028.

6. Lee TH, Hoover RL, Williams JD, Sperling RI, Ravalese Jr J, Spur BW, Robinson DR, Corey EJ, Lewis RA, Austen KF: Effect of dietary enrichment with eicosapentaenoic and docosahexaenoic acids on in vitro neutrophil and monocyte leukotriene generation and neutrophil function. $N$ Eng J Med 1985, 312:1217-1224.

7. Arisaka M, Arisaka O, Yamashiro Y: Fatty acid and prostaglandin metabolism in children with diabetes mellitus. II. The effect of evening primrose oil supplementation on serum fatty acid and plasma prostaglandin levels. Prostaglandin Leukot Essential Fatty Acids 1991, 43:197-201.

8. Kapoor R, Huang YS: Gamma linolenic acid: an antiinflammatory omega-6 fatty acid. Current Pharmaceut Biotech 2006, 7:531-534

9. Takai S, Jin D, Kawashima H, Kimura M, Shiraishi-Tateishi A, Tanaka T, kakutani S, Tanaka K, Kisa Y, Miyazaki M: Anti-atherosclerotic effects of dihomo-gamma-linolenic acid in ApoE-deficient mice. $J$ Atherosclerosis Thromb 2009, 16:480-489.

10. Mantzioris E, James MJ, Gibson RA, Cleland LG: Dietary substitution with an alpha-linolenic acid-rich vegetable oil increases eicosapentaenoic acid concentrations in tissues. Am J Clin Nutr 1994, 59:1304-1309.

11. Whelan J: Dietary stearidonic acid is a long chain $(n-3)$ polyunsaturated fatty acid with potential health benefits. J Nutr 2009, 139:5-10.

12. Department Health and Human Services N, NHLBI: Expert panel report 2: guidelines for the diagnosis and management of asthma. N/H Publication No $07-4051$ 2007. http://www.nhlbi.nih.gov/guidelines/asthma/asthgdln.pdf.

13. Johnson MM, Swan DD, Surette ME, Stegner J, Chilton T, Fonteh AN, Chilton FH: Dietary supplementation with gamma -linolenic acid alters fatty acid content and eicosanoid production in healthy humans. J Nutr 1997, 127:1435-1444.
14. Metcalfe LD, Schmitz AA, Pelka JR: Rapid preparation of fatty acid esters from lipids for gas chromatographic analysis. Anal Chem 1966, 38:514-515.

15. Weaver KL, Ivester P, Seeds MC, Case LD, Arm J, Chilton FH: Effect of dietary fatty acids on inflammatory gene expression in healthy humans. J Biol Chem 2009, 284:15400-15407.

16. Sloane DE, Tedla N, Awoniyi M, MacGlashan DW Jr, Borges L, Austen KF, Arm JP: Leukocyte immunoglobulin-like receptors: novel innate receptors for human basophil activation and inhibition. Blood 2004, 104:2832-2839.

17. Nauseef WM: Isolation of human neutrophils from venous blood. Meth Mol Biol 2007, 412:15-20.

18. Iversen $\mathrm{L}$, Fogh $\mathrm{K}$, Kragballe $\mathrm{K}$ : Effect of dihomogammalinolenic acid and its 15-lipoxygenase metabolite on eicosanoid metabolism by human mononuclear leukocytes in vitro: selective inhibition of the 5lipoxygenase pathway. Archives of Dermatol Res 1992, 284:222-226.

19. de Lorgeril M, Salen P: Alpha-linolenic acid and coronary heart disease. Nutr Metab Cardiovas Dis 2004, 14:162-169.

20. Wada M, DeLong CJ, Hong YH, Rieke CJ, Song I, Sidhu RS, Yuan C, Warnock M, Schmaier AH, Yokoyama C, et al: Enzymes and receptors of prostaglandin pathways with arachidonic acid-derived versus eicosapentaenoic acid-derived substrates and products. J Biol Chem 2007, 282:22254-22266.

21. Broughton KS, Johnson CS, Pace BK, Liebman M, Kleppinger KM: Reduced asthma symptoms with n-3 fatty acid ingestion are related to 5 -series leukotriene production. Am J Clin Nutr 1997, 65:1011-1017.

22. Kaur G, Cameron-Smith D, Garg M, Sinclair AJ: Docosapentaenoic acid (22:5n-3): a review of its biological effects. Prog Lipid Res 2011, 50:28-34.

23. Calder P: $n-3$ Polyunsaturated fatty acids and inflammation: from molecular biology to the clinic. Lipids 2003, 38:343-352.

24. Seyberth HW, Oelz O, Kennedy T, Sweetman BJ, Danon A, Frolich JC, Heimberg M, Oates JA: Increased arachidonate in lipids after administration to man: effects on prostaglandin biosynthesis. Clin Pharmacol Ther 1975, 18:521-529.

25. Barham JB, Edens MB, Fonteh AN, Johnson MM, Easter L, Chilton FH: Addition of eicosapentaenoic acid to $\mathrm{g}$-linolenic acid-supplemented diets prevents serum arachidonic acid accumulation in humans. J Nutr 2000, 130:1925-1931.

26. Surette ME, Koumenis IL, Edens MB, Tramposch KM, Chilton FH: Inhibition of leukotriene synthesis, pharmacokinetics, and tolerability of a novel dietary fatty acid formulation in healthy adult subjects. Clin Therapeut 2003, 25:948-971.

27. Surette ME, Koumenis IL, Edens MB, Tramposch KM, Clayton B, Bowton D, Chilton FH: Inhibition of leukotriene biosynthesis by a novel dietary fatty acid formulation in patients with atopic asthma: a randomized, placebocontrolled, parallel-group, prospective trial. Clin Therapeut 2003, 25:972-979.

28. Nouri-Aria KT, Irani AM, Jacobson MR, O'Brien F, Varga EM, Till SJ, Durham SR, Schwartz LB: Basophil recruitment and IL-4 production during human allergen-induced late asthma. J Allergy Clin Immunol 2001, 108:205-211.

29. Milgrom H, Fick RB, Su JQ, Reimann JD, Bush RK, Watrous ML, Metzger WJ: Treatment of allergic asthma with monoclonal anti-lgE antibody. N Eng J Med 1999, 341:1966-1973.

30. Arm JP, Lee TH: Sulphidopeptide leukotrienes in asthma. Clin Sci 1993, 84:501-510

31. Arm JP, Horton CE, Mencia-Huerta JM, House F, Eiser NM, Clark TJ, Spur BW, Lee TH: Effect of dietary supplementation with fish oil lipids on mild asthma. Thorax 1988, 43:84-92.

32. Arm JP, Horton CE, Spur BW, Mencia-Huerta JM, Lee TH: The effects of dietary supplementation with fish oil lipids on the airways response to inhaled allergen in bronchial asthma. Am Rev Respir Dis 1989, 139:1395-1400.

33. Lee TH, Mencia-Huerta JM, Shih C, Corey EJ, Lewis RA, Austen KF: Effects of exogenous arachidonic, eicosapentaenoic, and docosahexaenoic acids on the generation of 5-lipoxygenase pathway products by ionophoreactivated human neutrophils. J Clin Invest 1984, 74:1922-1933.

34. Calder PC: $\mathrm{n}-3$ Polyunsaturated fatty acids, inflammation, and inflammatory diseases. Am J Clin Nutr 2006, 83:S1505-S1519.

doi:10.1186/1476-511X-12-141

Cite this article as: Arm et al:: Impact of botanical oils on

polyunsaturated fatty acid metabolism and leukotriene generation in mild asthmatics. Lipids in Health and Disease 2013 12:141. 